\title{
Polarization strategies to improve the emission of Si-based light sources emitting at $1.55 \mu \mathrm{m}$
}

\author{
J.M. Ramírez ${ }^{\mathrm{a}, *}$, O. Jambois ${ }^{\mathrm{a}}$, Y. Berencén ${ }^{\mathrm{a}}$, D. Navarro-Urrios ${ }^{\mathrm{a}}$, A. Anopchenko ${ }^{\mathrm{b}}$, A. Marconi $^{\mathrm{b}}$, \\ N. Prtljaga ${ }^{\text {b }}$, N. Daldosso ${ }^{\text {d }}$, L. Pavesi ${ }^{\text {b }}$, J.-P. Colonna ${ }^{\text {c }}$, J.-M. Fedeli ${ }^{\mathrm{c}}$, B. Garrido ${ }^{\mathrm{a}}$ \\ a Departament d'Electrònica, Universitat de Barcelona, Carrer Martí i Franquès 1, Barcelona 08028, Spain \\ ${ }^{\mathrm{b}}$ Nanoscience Laboratory, Department of Physics, University of Trento, Via Sommarive 14, Povo (Trento) 38123, Italy \\ c CEA, Léti, Minatec Campus 17 rue des Martyrs, 38054 Grenoble Cedex 9, France \\ d Dipartimento di Informatica, Università di Verona, Strada Le Grazie 15, 37134 Verona, Italy
}

\section{A R T I C L E I N F O}

\section{Article history:}

Received 12 July 2011

Received in revised form 14 October 2011

Accepted 5 December 2011

Available online xxx

\section{Keywords:}

Silicon nanocrystals (Si-ncs)

Erbium

Pulsed voltage

Electroluminescence

\begin{abstract}
A B S T R A C T
We present a electroluminescence (EL) study of the Si-rich silicon oxide (SRSO) LEDs with and without $\mathrm{Er}^{3+}$ ions under different polarization schemes: direct current (DC) and pulsed voltage (PV). The power efficiency of the devices and their main optical limitations are presented. We show that under PV polarization scheme, the devices achieve one order of magnitude superior performance in comparison with DC. Time-resolved measurements have shown that this enhancement is met only for active layers in which annealing temperature is high enough $\left(>1000^{\circ} \mathrm{C}\right)$ for silicon nanocrystal (Si-nc) formation. Modeling of the system with rate equations has been done and excitation cross-sections for both $\mathrm{Si}$-nc and $\mathrm{Er}^{3+}$ ions have been extracted.
\end{abstract}

(c) 2011 Elsevier B.V. All rights reserved.

\section{Introduction}

Silicon based light sources have been widely investigated for their implementation in integrated photonic circuits [1-8]. Complementary metal-oxide-semiconductor (CMOS) compatibility would allow for cheap mass-fabrication and for electronic-photonic function integration in the same chip. In particular, the fabrication of an efficient electrically pumped source working in the near infrared would be of a great interest due to its applications in telecommunications [9]. With such an idea, $\mathrm{Er}^{3+}$ doped materials were fabricated and embedded in MOS devices to develop Si-based sources emitting at $1.54 \mu \mathrm{m}[10,11]$. It was demonstrated later that the inclusion of Si-ncs in $\mathrm{Er}^{3+}$ doped silica resulted in efficient room-temperature $\mathrm{Er}^{3+}$ luminescence under optical pumping [12-15]. The much larger effective absorption cross-section of Si-ncs with regard to $\mathrm{Er}^{3+}$ ions and the efficient coupling between them was an attractive perspective for the near future. Moreover, higher excitation cross-sections were measured in both species under electrical pumping [16,17], giving a room for improvement in these devices. However, the role of Si-ncs in $\mathrm{Er}^{3+}$ doped silica under electrical pumping is not

\footnotetext{
* Corresponding author. Tel.: +34 934039175.

E-mail address: jmramirez@el.ub.es (J.M. Ramírez).
}

fully resolved yet, as the excitation mechanisms and the physics involved are different from the optical pumping.

In order to shed light on this issue, Si-based light emitting devices (LEDs) with undoped Si-ncs (emitting in the visible) or doped with $\mathrm{Er}^{3+}$ ions (emitting in the near infrared) have been fabricated. The contribution of Si-ncs to the electro-optical properties and the influence of the annealing treatment for visible and infrared devices are reported, as well as the main excitation mechanisms involved in the electroluminescence. Moreover, PV studies have allowed us to investigate the EL response with the driving frequency, determining an optimized frequency of operation to maximize the EL of Si-ncs in more than one order of magnitude with respect to the electroluminescence under DC excitation.

\section{Experimental}

Three different MOS capacitors were fabricated on P-type silicon wafers doped at $1 \times 10^{15} \mathrm{at} / \mathrm{cm}^{3}$. The oxide was replaced by an optically active layer. A SRSO layer $50 \mathrm{~nm}$ thick was deposited by Low Pressure Chemical Vapor Deposition (LPCVD) with two different silicon excess (9\% and 16\%). Layers were annealed for $1 \mathrm{~h}$ at $900^{\circ} \mathrm{C}$ (D1 and D3) or at $1100^{\circ} \mathrm{C}$ (D2) to induce the phase separation of the $\mathrm{SRSO}$ in $\mathrm{Si}$ and $\mathrm{SiO}_{2}$. $\mathrm{An}^{3+}{ }^{3+}$ implantation was performed in one sample (D3), with a fluence of $1 \times 10^{15} \mathrm{at} / \mathrm{cm}^{2}$ and $25 \mathrm{keV}$ of energy. $\mathrm{Er}^{3+}$ ions were activated by a post implantation annealing performed at $800^{\circ} \mathrm{C}$ for $6 \mathrm{~h}$. The implantation profile and the Si 
Table 1

Fabrication parameters of the devices under study.

\begin{tabular}{|c|c|c|c|c|}
\hline Device & $\mathrm{Si}(\%)$ & Ann. temp. $(1 \mathrm{~h})$ & $\mathrm{Er}^{3+}$ implant. (25 keV) & Post-ann. temp. (6h) \\
\hline D1 & 9 & $900^{\circ} \mathrm{C}$ & - & - \\
\hline D2 & 16 & $1100^{\circ} \mathrm{C}$ & - & - \\
\hline D3 & 9 & $900^{\circ} \mathrm{C}$ & $10^{15} \mathrm{at} / \mathrm{cm}^{2}$ & $800^{\circ} \mathrm{C}(6 \mathrm{~h})$ \\
\hline
\end{tabular}

excess were further confirmed by Secondary Ion Mass Spectroscopy (SIMS) and X-ray Photoelectron Spectroscopy (XPS). Details on the procedure and results can be found in Ref. [18]. Finally, a highly n-type doped $\left(1 \times 10^{20} \mathrm{at} / \mathrm{cm}^{3}\right)$ polysilicon layer $100 \mathrm{~nm}$ thick was deposited and used as a semitransparent gate electrode. The area of devices is $0.09 \mathrm{~mm}^{2}$. The details of each of the three layers are resumed in Table 1.

EL measurements were performed at room temperature. LEDs were biased under forward condition, i.e. negative voltages over the gate, with a probe station (Cascade Microtech Summit 1100) driven either by a semiconductor device analyzer (Agilent B1500A) for DC measurements, or by a square pulse generator for pulsed excitation (Agilent 8114A).

EL was collected either with a photomultiplier tube (PMT) or with a cryogenically cooled charged coupled device (CCD), placed behind an Acton 2300i grating spectrometer for spectra recording. A digital oscilloscope was used for time resolved measurements. All the spectra were corrected from the spectral response.

\section{Results}

\subsection{Power efficiency}

The electro-optical characterization of the devices was done by polarizing them from low voltages until breakdown, collecting at the same time the leakage current through the gate and the emitted EL. High electric fields of around $10 \mathrm{MV} / \mathrm{cm}$ at the maximum were reached.

Fig. 1 shows the comparison of the power efficiency $(\eta)$ between visible (a) and infrared (b) emitting LEDs as a function of the DC voltage (forward condition). It is defined as the ratio between the collected optical power and the applied input power:

$\eta=\frac{\text { Opt. power }(w)}{\text { Elect. power }(w)} \times 100$

obtaining a maximum of $\eta_{\mathrm{D} 2}=4 \times 10^{-4} \%$ in the visible range and $\eta_{\mathrm{D} 3}=10^{-2} \%$ in the infrared. A decrease of the power efficiency takes place in both devices when the voltage is increased. Nonetheless, this latter fact can be controlled for visible LEDs (see Fig. 1a) by decreasing both the amount of Si excess in the matrix and the annealing temperature, achieving a wide range of voltages (from $17 \mathrm{~V}$ to $35 \mathrm{~V}$ ) where the power efficiency remains almost constant, but at lower values. This suggests the existence of a compromise between the power efficiency and its non-dependence on the voltage in such systems.

The threshold voltage of electroluminescence $\left(\mathrm{V}_{\mathrm{TH}}\right)$ can be extracted from the insets of Fig. $1 \mathrm{a}$ (16V for D1 and $21 \mathrm{~V}$ for D2) and Fig. $1 \mathrm{~b}$ ( $26 \mathrm{~V}$ for $\mathrm{D} 3)$, where the EL has been represented as a function of the applied DC voltage in arbitrary units. For visible emitting LEDs, the threshold of the EL is directly correlated with the annealing temperature, as smaller $V_{\mathrm{TH}}$ are obtained when the annealed temperature is lower. However, no substantial difference was observed in devices with the same annealing treatment but different Si excess (9\% and 16\%). Regarding the Si-nc size, although TEM measurements were not accomplished in the samples annealed at $900^{\circ} \mathrm{C}$ and at $1100^{\circ} \mathrm{C}$, similar EL spectral shapes centered at the same wavelength were obtained for devices D1 and D2, suggesting similar mean Si-nc diameters in both samples regardless of the applied annealing temperature $\left(900^{\circ} \mathrm{C}\right.$ or $1100^{\circ} \mathrm{C}$ ). Moreover, the size of the $\mathrm{Si}$-nc was roughly estimated considering the theoretical results of Delerue et al., where the emitted $\lambda$ is correlated with the Si-nc diameter as follows [19]:

$E=1.12+\frac{3.73}{d^{1.39}}$

where $E$ is the emission energy and d the diameter of the Si-nc. A mean value of $(4.7 \pm 0.3) \mathrm{nm}$ was found for the sample annealed at $900^{\circ} \mathrm{C}$, and $(4.5 \pm 0.3) \mathrm{nm}$ for the one annealed at $1100^{\circ} \mathrm{C}$. Although these results seem to be contradictory with the well establish belief that the Si-nc growth is expected to increase with the temperature, it is important to remark that at the lowest temperature, i.e. $900{ }^{\circ} \mathrm{C}$, there is no significant crystallization of Si nanoparticles [20] and hence they grow as amorphous Si clusters. This assumption is reinforced when considering the quantum confinement of e-h pairs
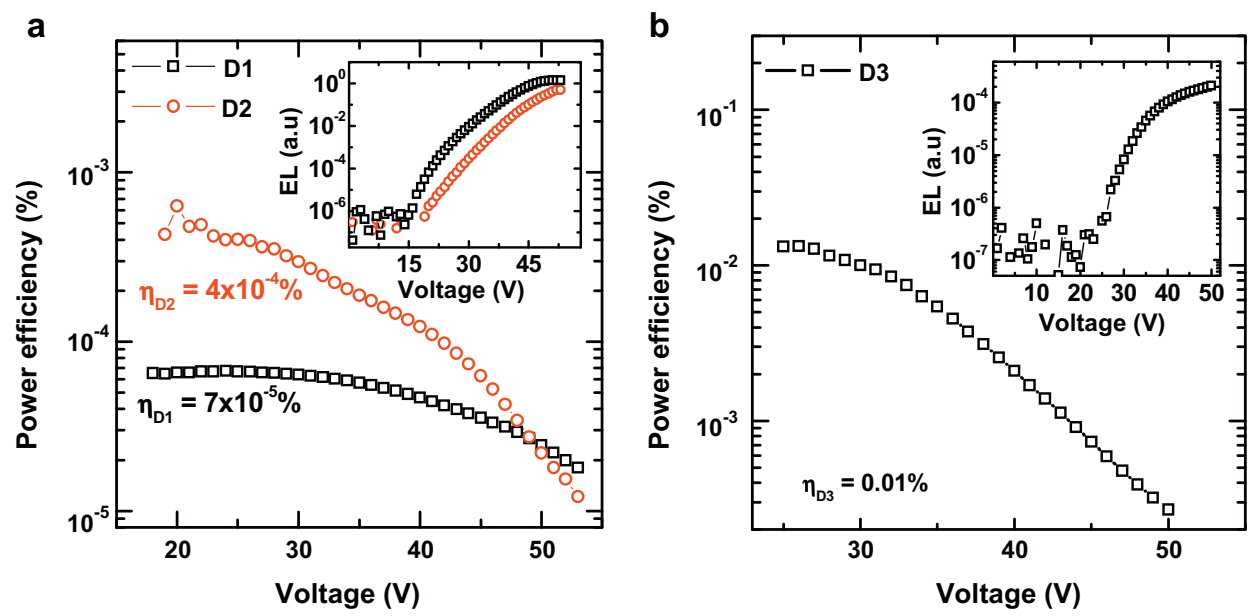

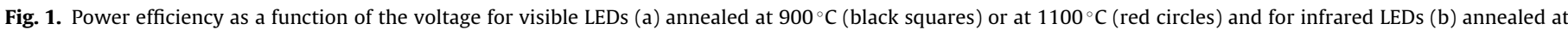

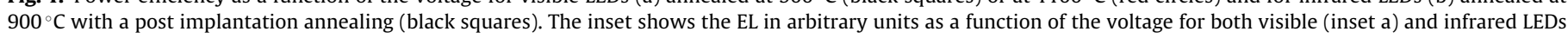
(inset b). (For interpretation of the references to color in this figure legend, the reader is referred to the web version of this article.) 

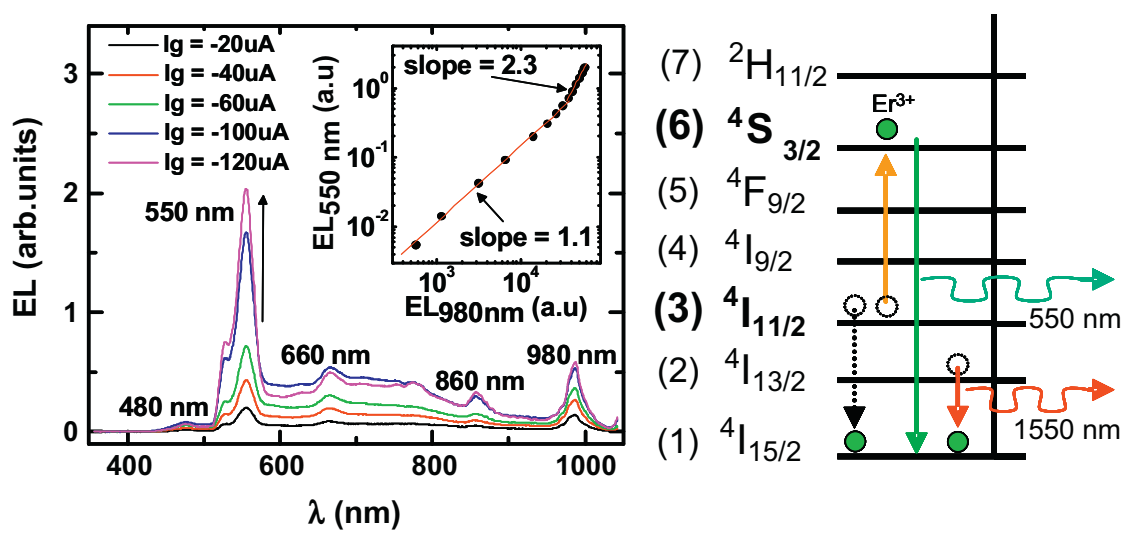

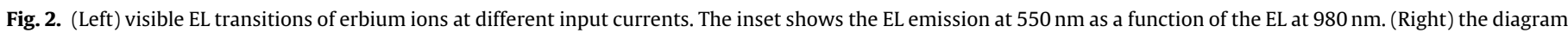

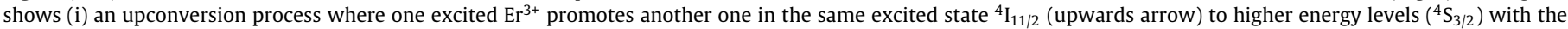
following emission at $550 \mathrm{~nm}$; (ii) $\mathrm{Er}^{3+}$ emission at $1550 \mathrm{~nm}$ from the first excited level $\left({ }^{4} \mathrm{I}_{13 / 2}\right)$ to the ground state $\left({ }^{4} \mathrm{I}_{15 / 2}\right)$.

inside these nanocomposites [21], since the comparison of the EL spectra for D1 and D2 under the same current density $\left(10 \mathrm{~mA} / \mathrm{cm}^{2}\right)$ provides higher EL intensity (5 times) in the sample annealed at $1100^{\circ} \mathrm{C}$.

In addition, the comparison between D1 (visible) and D3 (infrared) with an extra annealing treatment at $800^{\circ} \mathrm{C}$ for $6 \mathrm{~h}$ shows a clear shift in the $V_{\mathrm{TH}}$ of about $10 \mathrm{~V}$, suggesting that the erbium implantation and the post-implantation annealing treatment remarkably affect the EL(V) characteristics.

By other side, the saturation of the EL at high electric fields (above $8 \mathrm{MV} / \mathrm{cm}$ ) in both devices is ascribed to a saturation of the number of excited luminescent centers and/or quenching of the additional excitation by the increasing amount of current. The quantification of the inverted fraction of excited luminescent centers was done for the infrared (D3), obtaining values around $20 \%$ for this sample, equally than Ref. [14].

\subsection{Limiting factors of $\mathrm{Er}^{3+}$ ions}

Focusing on infrared emitting LEDs (D3), we observed that one of the main limiting mechanisms involved in the emission of the first excited level of $\mathrm{Er}^{3+}$ when pumping at high current densities (above $100 \mathrm{~mA} / \mathrm{cm}^{2}$ ) was the cooperative up-conversion. The excitation of an $\mathrm{Er}^{3+}$ ion from one transition state to another one with higher energy as a consequence of the interaction with a neighboring erbium becomes critical at high fluxes [22]. This effect was experimentally proved in our devices by collecting the visible spectrum when the polarization current applied is increased, as can be observed in Fig. 2. At low input currents, the EL of every transition level increases linearly with respect to the other ones. However, at a certain current, the excited level ${ }^{4} \mathrm{~S}_{3 / 2}$ becomes overpopulated by the ${ }^{4} \mathrm{I}_{11 / 2}$ level, enhancing the EL of the transition ${ }^{4} \mathrm{~S}_{3 / 2} \rightarrow{ }^{4} \mathrm{I}_{15 / 2}$ and diminishing the decay times involved in the first excited transition. Therefore, a sudden slope change occurs when the EL at $550 \mathrm{~nm}\left({ }^{4} \mathrm{~S}_{3 / 2} \rightarrow{ }^{4} \mathrm{I}_{15 / 2}\right)$ is plotted as a function of the EL at $980 \mathrm{~nm}$ $\left({ }^{4} I_{11 / 2} \rightarrow{ }^{4} I_{15 / 2}\right)$, as observed in the inset of Fig. 2 . Moreover, the emission at $1.55 \mu \mathrm{m}$ saturates at these high fluxes, whereas the emission at $550 \mathrm{~nm}$ becomes quadratic with the current until the breakdown of the device.

On the other hand, the existence of a threshold voltage (26V) to obtain simultaneously the infrared EL in addition with the visible EL peaks attributed to higher $\mathrm{Er}^{3+}$ transitions points out that carriers have energies ranged from $0.8 \mathrm{eV}$ (EL at $1550 \mathrm{~nm}$ ) to $2.5 \mathrm{eV}$ (EL at $488 \mathrm{~nm}$ ) from the very beginning of the EL [10]. Then, considering the EL spectra of the $\mathrm{Er}^{3+}$ doped $\mathrm{SiO}_{2}$ free of Si-ncs [23] and taking into account that the energy transfer rates occurs at lower energy values [24], we ascribe the $\mathrm{Er}^{3+}$ excitation to the direct impact of hot carriers highly accelerated. This idea was further corroborated by studying the transport mechanisms prevailing inside layers with two well known models [25]; (i) Poole-Frenkel conduction of thermally activated carriers; (ii) Fowler-Nordheim tunneling of hot carriers through a triangular barrier height. Therefore, the fit of these models over the $I(V)$ curves of visible and infrared LEDs determined that at low-moderate voltages, in which the EL does not show up, the main transport occurs through trap assisted centers (Poole-Frenkel), whereas at high voltages the Fowler-Nordheim mechanism would be the responsible of the conduction, coinciding with the EL emission [26].

\subsection{Pulsed voltage excitation and time-resolved measurements}

In order to study the excitation and de-excitation properties involved in the EL, LEDs were polarized under a negative square pulsed voltage (from 0 to $-30 \mathrm{~V}$ ). The driving frequency was swept from $10 \mathrm{~Hz}$ to $1 \mathrm{MHz}$. Results for visible emitting LEDs (D1 and D2) are shown in Fig. 3a. Each point corresponds to an integrated EL spectrum. The comparison between the frequency evolution of the EL in D1 (black squares) and D2 (red circles) under a negative square pulse with the same voltage than the one applied in the DCEL (black dashed line and red dotted line) reveals a clear difference between them. Whereas the emission of the D1 does not show an improvement with regard to the DC value, the EL of D2 becomes increased above the DC value of about one order of magnitude at $20 \mathrm{kHz}$. Note that the inverse of this value coincides with the experimental lifetime obtained from Si-ncs $(50 \mu \mathrm{s})$ [27]. This EL improvement was quantified by diminishing the input voltage in order to obtain comparable EL values with the ones provided by the DC polarization, determining a diminution of $5 \mathrm{~V}$ in the input $\mathrm{AC}$ voltage (from 0 to $-25 \mathrm{~V}$, blue triangles in Fig. 3a) with regard to the same EL in DC (at $-30 \mathrm{~V})$.

In addition, time-resolved measurements were carried out in D1 and D2 so as to understand the frequency evolution of the visible EL under PV excitation. Looking at the device D2, a transient EL overshoot is observed when the polarization is switched off (Fig. 3b). On the contrary, no EL overshoot arises when biasing D1 under the same polarization conditions. Therefore, the enhancement of the EL intensity under PV excitation observed in device D2 (Fig. 3a, red empty circles) is attributed to the sum of this EL overshoot with the DC value of the EL (shown in Fig. 3b). We associate the presence of the overshoot in sample D2 with the thermal treatment of the sample. If an adequate annealing temperature is performed, the crystallization of Si-ncs occurs and hence the created excitons 
a

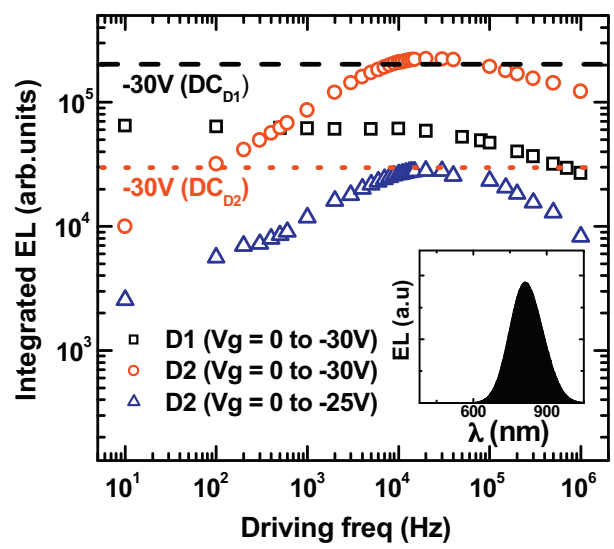

b

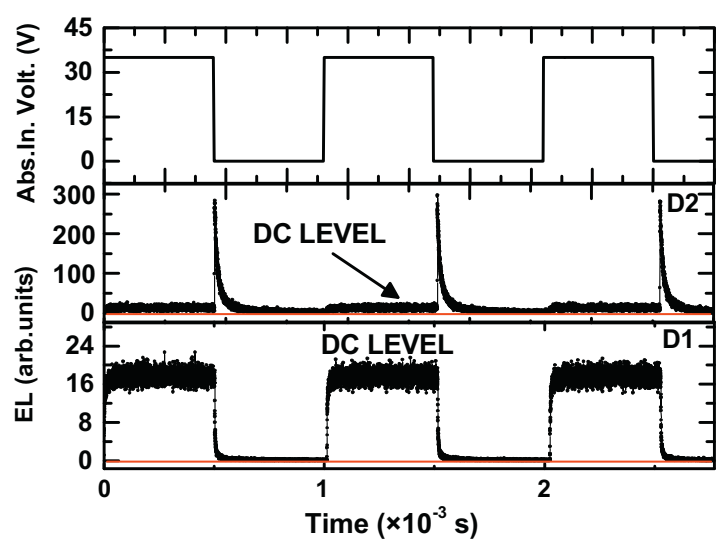

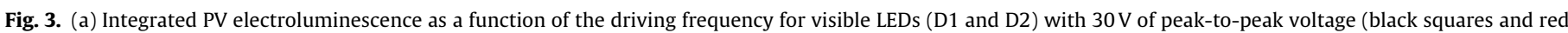

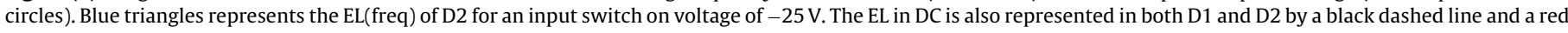

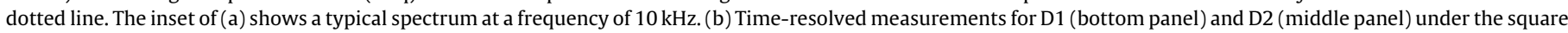

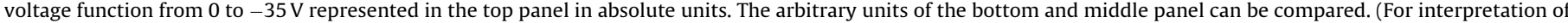
the references to color in this figure legend, the reader is referred to the web version of this article.)

remain inside the Si-ncs even after the voltage switch off. At this moment, the wave function overlap of each confined e-h pair is maximized and consequently the probability of radiative recombination is increased [28]. Furthermore no Auger processes due to injected hot carriers would be involved during the off voltage state [29], obtaining an EL enhancement of one order of magnitude compared to the DC level of the EL. On the contrary, applying an insufficient annealing temperature no Si crystallization takes place, providing a weak excitonic confinement inside them and stimulating the exciton migration out of the Si-nc. This hypothesis was further supported by comparing the lifetimes in samples D1 and D2 [30]. A difference of 10 times was detected between the sample D1 $(\sim 3 \mu \mathrm{s})$ and sample D2 $(\sim 30 \mu \mathrm{s})$. Thus, these results strengthen the importance of the annealing temperature in these LEDs.

For infrared LEDs (D3), no improvement of the EL at $1.55 \mu \mathrm{m}$ was detected under AC compared with the DC excitation. The evolution of the integrated EL as a function of the driving frequency and time resolved measurements were similar than D1, without EL overshoot. However, in this case, the lack of improvement of the infrared signal cannot be related to the non-crystallization of Sincs, as we have shown that they act just as conduction centers for hot carriers and not as sensitizers. In our layers, $\mathrm{Er}^{3+}$ ions would be directly excited with hot electrons. This suggests that Auger processes above mentioned occurring in the Si-ncs are not affecting the $\mathrm{Er}^{3+}$ ions significantly.

From Fig. 3, the absorption cross-section was estimated in visible (D1) and infrared (D3) LEDs taking into account a nearly two level system, which has been used by many authors before [31,32]. Starting from the dynamic rate equation,

$\frac{d N_{2}}{d t}=\sigma \frac{j}{e}\left(N_{\text {total }}-N_{2}\right)-\frac{N_{2}}{\tau_{\text {decay }}}$

with

$\frac{1}{\tau_{\text {decay }}}=\frac{1}{\tau_{\text {rad }}}+\frac{1}{\tau_{\text {non-rad }}}$

where $\sigma$ is the effective absorption cross-section, $j$ is the incident current density, $e$ the single electron charge, $\tau_{\text {decay }}$ is the total lifetime (radiative $\left(\tau_{\text {rad }}\right)$ and non-radiative $\left(\tau_{\text {non-rad }}\right)$ ), and $N_{\text {total }}$ and $\mathrm{N}_{2}$ the total implanted and the excited $\mathrm{Er}^{3+}$ ions in the first energy
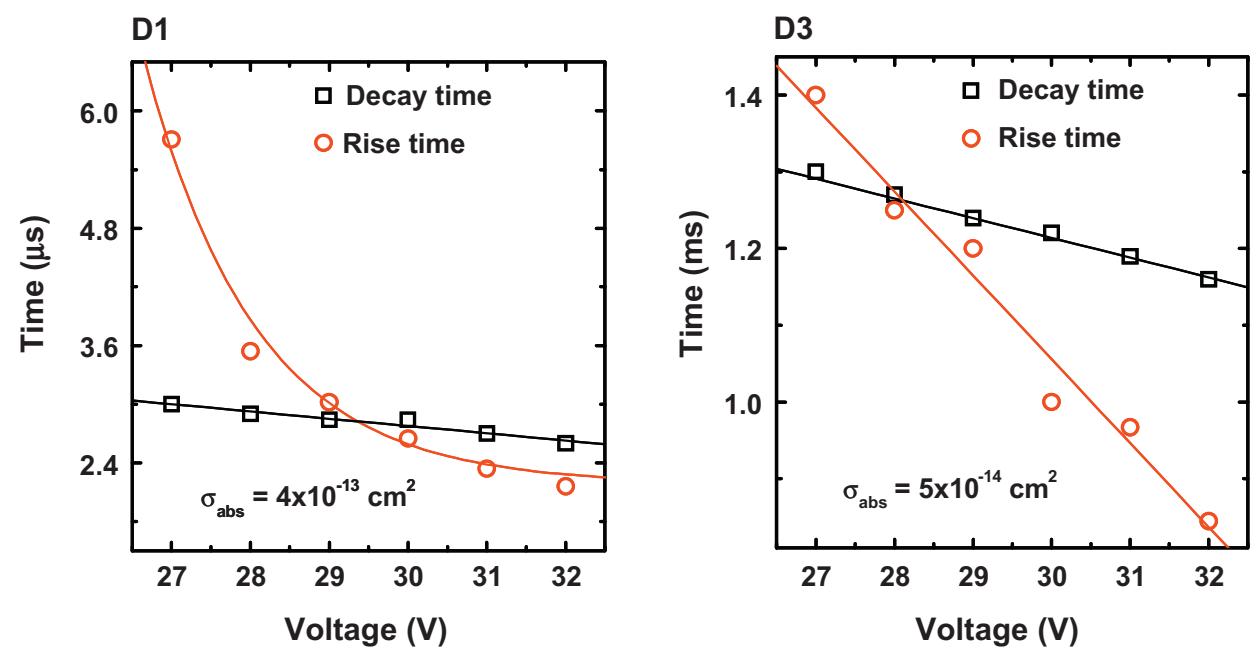

Fig. 4. Experimental decay and rise times as a function of the applied voltage for visible (a) and infrared (b) LEDs. Fitted lines are guides to the eye. 
level, respectively. Also, knowing that $\mathrm{EL} \propto N_{2} / \tau_{\text {decay }}$, the following relation can be obtained [31],

$\frac{1}{\tau_{\text {rise }}}=\frac{1}{\tau_{\text {decay }}}+\frac{j}{e} \sigma$

being $\sigma$ the absorption cross-section of the luminescent centers. A single exponential was used to fit the decay in the $\mathrm{Er}^{3+}$ doped sample. For Si-ncs, a stretched exponential decay is typically reported in the literature [33],

$\mathrm{EL}(t)=\mathrm{EL}_{0} \exp \left[-\left(\frac{t}{\tau_{\text {decay }}}\right)^{\beta}\right]$ with $\beta<1$

Fig. 4 shows the decay lifetime ( $\tau_{\text {decay }}$, black squares) and the rise time ( $\tau_{\text {rise }}$, red circles) as a function of the applied voltage, under a negative square pulsed polarization at very low frequencies $(10 \mathrm{~Hz})$. Notice that both lifetimes decrease with the voltage bias, regardless of the emission range of the devices (visible or infrared emitting LEDs). Also, the rise time observed is higher than the decay time at low voltages (below $30 \mathrm{~V}$ ), which has been ascribed to charge transport among Si-ncs, suggesting that the absorption cross-section cannot be calculated as a nearly two level system in this range (see Eq. (5)). Nonetheless, Eq. (5) has been used to estimate the absorption cross sections from $30 \mathrm{~V}$ and above, where $\tau_{\text {decay }}>\tau_{\text {rise }}$ obtaining a $\sigma_{\text {abs }}=4 \times 10^{-13} \mathrm{~cm}^{2}$ with a $\beta=0.8$ for Sincs and $\sigma_{\mathrm{abs}}=5 \times 10^{-14} \mathrm{~cm}^{2}$ for $\mathrm{Er}^{3+}$ ions. Therefore, the absorption cross-section of Si-ncs is 8 times higher than the one of erbium ions, in agreement with previous works [32], which have reported a difference of 5 times between them.

\section{Conclusions}

We have studied the EL properties of LPCVD SiO ${ }_{x}$ layers doped or not with $\mathrm{Er}^{3+}$ ions, under DC and pulsed polarization. Under DC excitation, we show that annealing temperature affects both power efficiency and its dependence with voltage. In addition, the annealing treatment has been demonstrated to be an important parameter to enhance the EL properties under pulsed polarization. In the visible, an increase of the EL-frequency characteristics is observed for the layers having well crystallized Si nanoclusters. This results in a well defined EL overshoot appearing when the pulsed voltage is in off state and attributed to the suppression of Auger effect in the Si-nc and to the e-h wave function overlap, allowing the radiative recombination of the remaining excitons. However, no EL improvement was observed for $\mathrm{Er}^{3+}$ doped layers. Moreover, the excitation of $\mathrm{Er}^{3+}$ ions has been ascribed to direct impact excitation.

\section{Acknowledgements}

This work is supported by HELIOS Project (FP7 224312) and ITO9ILB2F9 (Integrated Italia-Spain Action) Project.

\section{References}

[1] G. Franzò, A. Irrera, E.C. Moreira, M. Miritello, F. Iacona, D. Sanfilippo, G. Di Stefano, P.G. Fallica, F. Priolo, Appl. Phys. A 74 (2002) 1.

[2] L. Pavesi, L. Dal Negro, C. Mazzoleni, G. Franzò, F. Priolo, Nature 408 (2000) 440.

[3] A. Pérez-Rodríguez, O. González-Varona, B. Garrido, P. Pellegrino, J.R. Morante, J. Appl. Phys. 94 (2003) 254.

[4] M.V.Wolkin, J.Jorne, P.M. Fauchet, G. Allan, C. Delerue, Phys. Rev. Lett. 82 (1999) 197.

[5] K. Cho, N. Park, T. Kim, K. Kim, G. Sung, J. Shin, Appl. Phys. Lett. 86 (2005) 71909.

[6] L. Dal Negro, J. Yi, L. Kimerling, S. Hamel, A. Williamson, G. Galli, Appl. Phys. Lett. 88 (2006) 233109

[7] O. Jambois, Y. Berencén, K. Hijazi, M. Wodjak, A.J. Kenyon, F. Gourbilleau, R. Rizk, B. Garrido, J. Appl. Phys. 106 (2009) 63526.

[8] Y. Berencén, J. Carreras, O. Jambois, J.M. Ramírez, J.A. Rodríguez, C. Domínguez, C.E. Hunt, B. Garrido, Opt. Express 19 (2011) A234.

[9] A. Polman, J. Appl. Phys. 82 (1997) 1.

[10] S. Coffa, G. Franzò, F. Priolo, A. Pacelli, A. Lacaita, Appl. Phys. Lett. 73 (1998) 93.

[11] G.T. Reed, A.K. Kewell, Mater. Sci. Eng. B 40 (1996) 207.

[12] M. Fujii, M. Yoshida, Y. Kanzawa, S. Hayashi, K. Yamamoto, Appl. Phys. Lett. 71 (1997) 1198.

[13] H.-S. Han, S.-y. Seo, J.H. Shin, Appl. Phys. Lett. 79 (2001) 4568.

[14] O. Jambois, F. Gourbilleau, A.J. Kenyon, J. Montserrat, R. Rizk, B. Garrido, Opt. Express 18 (2010) 3.

[15] D. Navarro-Urrios, A. Pitanti, N. Daldosso, F. Gourbilleau, R. Rizk, B. Garrido, L. Pavesi, Phys. Rev. B 79 (2009) 193312.

[16] A. Irrera, D. Pacifici, M. Miritello, G. Franzò, F. Priolo, F. Iacona, D. Sanfilippo, G. Di Stefano, P.G. Fallica, Appl. Phys. Lett. 81 (2002) 1866.

[17] M.E. Castagna, S. Coffa, M. Monaco, A. Muscara, L. Caristia, S. Lorenti, A. Messina, Mater. Sci. Eng. B 105 (2003) 83.

[18] N. Prtljaga, D. Navarro-Urrios, A. Marconi, A. Anopchenko, J.-P. Colonna, F. Milesi, N. Daldosso, O. Jambois, B. Garrido, J.M. Fedeli, L. Pavesi, Opt. Mater. 33 (2011) 1083.

[19] C. Delerue, G. Allan, M. Lannoo, Phys. Rev. B 48 (1993) 11024.

[20] F. Iacona, C. Bongiorno, C. Spinella, S. Boninelli, F. Priolo, J. Appl. Phys. 95 (2004) 3723.

[21] O. Jambois, H. Rinnert, X. Devaux, M. Vergnat, J. Appl. Phys. 100 (2006) 123504.

[22] R. Kapoor, C.S. Friend, A. Biswas, P.N. Prasad, Opt. Lett. 25 (2000) 5

[23] Rare-Earth Implanted MOS Devices for Silicon Photonics, first ed., Springer Series in Materials Science, Springer, 2010.

[24] I. Izeddin, D. Timmerman, T. Gregorkiewicz, A.S. Moskalenko, A.A. Prokofiev, I.N. Yassievich, M. Fujii, Phys. Rev. B 78 (2008) 035327.

[25] X.D. Pi, O.H.Y. Zalloum, A.P. Knights, P. Mascher, P.J. Simpson, J. Phys. Condens. Matter 18 (2006) 9943-9950.

[26] O. Jambois, J.M. Ramírez, Y. Berencén, D. Navarro-Urrios, P. Pellegrino, A. Anopchenko, A. Marconi, N. Prtljaga, A. Tengattini, N. Daldosso, L. Pavesi, J.-P. Colonna, J.-M. Fedeli, B. Garrido, J. APD, in press.

[27] J. Valenta, N. Lalic, J. Linnros, Appl. Phys. Lett. 84 (2004) 9.

[28] C. Delerue, G. Allan, M. Lannoo, Phys. Rev. B 64 (2001) 193402.

[29] A. Irrera, F. Iacona, I. Crupi, C.D. Presti, G. Franzò, C. Bongiorno, D. Sanfilippo, G. Di Stefano, A. Piana, P.G. Fallica, A. Canino, F. Priolo, Nanotechnology 17 (2006) 1428-1436.

[30] S. Gardelis, A.G. Nassiopoulou, N. Vouroutzis, N. Frangis, J. Appl. Phys. 105 (2009) 113509.

[31] F. Priolo, G. Franzò, D. Pacifici, V. Vicinguerra, F. Iacona, A. Irrera, J. Appl. Phys. 89 (2001) 264.

[32] F. Iacona, D. Pacifici, A. Irrera, M. Miritello, G. Franzò, F. Priolo, D. Sanfilippo, G. Di Stefano, P.G. Fallica, Appl. Phys. Lett. 81 (2002) 3242.

[33] J. Linnros, N. Lalic, A. Galeckas, V. Grivickas, J. Appl. Phys. 86 (1999) 6128. 